\title{
Effect of electric and magnetic field on welding parameters in plasma welding
}

\author{
R. K. Tyagi ${ }^{1 *}$, R. S. Pandey ${ }^{2}$, Arbind Kumar ${ }^{3}$, K. K. Srivastava ${ }^{4}$

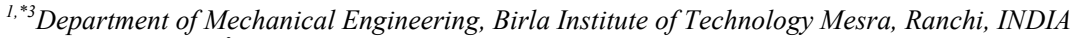 \\ ${ }^{3}$ Department of Physics, Amity University Noida, INDIA \\ ${ }^{4}$ Central University Ranchi Jharkhand, INDIA \\ "Corresponding Author: e-mail: tyagi_rk1@rediffmail.com, Tel+91-9911393047
}

\begin{abstract}
Plasma welding parameters have been systemically investigated by using experimental factors/constraints along with numerical analysis and the help of mathematical formulation as well as computer technique. In this paper, KSF6 gas has been used to generate plasma which contains velocity shear instability. The plasma welding parameters like Debye length, temperature of ions and the number of ions has been examined by taking experimental parameters. The results obtained by theoretical calculations are identical to the experimental results. In this work, influences of electric and magnetic field on Debye length, temperature of ions and the number of ions have been quantified. Theoretical investigation on one hand, while plasma welding parameters on the other increased by increasing the values of homogenous DC electric field. It also decreased by increasing the value of magnetic field. The controlling of welding parameters by electric and magnetic field has been discussed.
\end{abstract}

Keywords: Plasma, Velocity Shear Instability, Welding parameters, Plasma welding.

DOI: http://dx.doi.org/10.4314/ijest.v3i8.14

\section{Introduction}

The plasma welding process was introduced to the welding industry in 1964 as a method of bringing better control to the arc welding process in lower current ranges. Today, plasma retains the original advantages it brought to the industry by providing an advanced level of control and accuracy to produce high quality weld in both miniature and precision applications and to provide long electrode life for high production requirements at all levels of amperage. It is used in a variety of joining operations ranging from welding of miniature components to seam welding, to high volume production welding, and many others (Bernard and Heinzman., 1999). Plasma welding is similar to Gas Tungsten Arc Welding. The significant difference between the two is that the arc plasma is constricted by a nozzle to produce a high-energy plasma stream in which temperatures between $10,000^{\circ} \mathrm{C}$ and $20,000^{\circ} \mathrm{C}$ are attained. Since the plasma jet is extremely narrow, it can not provide adequate protection for the weld pool, which is why it is necessary to add a large diameter annular stream of shielding gas (Tse et al., 1999). In the process of deep penetration welding with a laser, a partially ionized plasma forms around the focal point of the laser light. This laser-induced plasma significantly affects the efficiency of deep penetration laser welding due to its shielding effect. A lot of research has been done in the last few decades primarily to the control of plasma. The most successful method developed so far is to disrupt the plasma by using a side jet of helium. Some newer methods which are different from the traditional plasma-control techniques have been recently proposed. Liu and Tse have tried to use the effect of magnetic field to reduce the shielding effect of the plasma (Tse et al., 2000).

The application of structures with Aluminium alloys in different areas of industry is continuously increasing. The fabrication and repair of these structures are carried out by a number of welding technologies, in particular, argon-shielded electrode welding both consumable and non-consumable. The application of arc technologies is accompanied by a number of issues, addressed by both the special features of welding materials and by limited possibilities of these methods. They include the low productivity in welding 
metals of increased thickness, defectiveness of welded joints, the heterogeneity of the welded joints and parent metal and problems in the fabrication of large structures with different spatial distribution of the welded joints. Characteristic defects in arc welded Aluminium alloys are gas porosity, formation of oxide films, tungsten and other inclusions, hot and cold cracks, lack of fusion, defects, cavities, etc. The majority of these problems can be solved and productivity can be increased by plasma welding (Shchitsyn ans Shchitsyn, 2003). Litovoko (2008) presented a model in which charged particle beam is generated by an electric and magnetic fields, and considering collision-less plasma for homogeneously distributed charged particles. Tyagi et al. (2011) presented a model of metal cutting. The gas used to melt the metal was KSF6 based on the concept of velocity shear instability.

Plasma jet can be regarded as a multi functional fluid, since it has high enthalpy, chemical reactivity and is easily controllable. It has been extensively utilized in the field of metallurgical and chemical processing, propulsion for space vehicles and MHD generation. In these industrial applications, it is very important to control precisely the characterization of a plasma jet from both micro and macro point of view. Nishiyama et al. (1992) investigated the effect of magnetic field on the thermo fluid characteristic of heavy particles and plasma parameters such as plasma species number density and temperature in plasma jet.

The plasma generation by a coaxial plasma puff-gun using a double Langmuir probe and spectrometer has been studied by Shen et al. (1995). Details of plasma parameters, i.e. change in voltage of energy storage capacitors which ranges from 1.5 to $4.0 \mathrm{KV}$, the plasma electron temperature from 10 to $20 \mathrm{eV}$, the kinetic energy from 45 to $310 \mathrm{eV}$ and the density from $5 \times 10^{13}$ to $7 \times 10^{14}$ $\mathrm{cm}^{3}$ has also been discussed. An approximate solution has been obtained under the assumption of the snowplow model, and the comparison of the predictions of the theory with our experimental results indicates general qualitative agreement.

The aim of this work was to find out the effect of magnetic field and electric fields on Debye length, temperature of ions, and number of ions striking the probe, by using mathematical expressions and computer technique. Heat transfer from plasma to work piece depends on many parameters. Temperature of ions, Debye length, and number of ions striking the probe are also some important factors (Bychenkon and Silin, 1981). This study will be useful in understanding the heat transfer between plasma and electrode and this phenomenon can be altered in an existing plasma welding machine by changing the electric and magnetic fields.

\section{Working and model description}

\subsection{Working}

The working principle of the model described here is similar to that indicated in Tyagi and Pandey (2011) in which plasma with velocity shear instability is used to melt the metal to be welded. It should be noted that by forcing the plasma with velocity shear instability through a constricted orifice, the torch transmits a high concentration of heat over a small area. Consequently, with high performance welding equipment, the plasma process produces exceptionally high quality weld. Note that plasma gas is $\mathrm{K}+$ and SF6- ions. Also, note that the torch uses a secondary gas which can be argon, helium, etc, which assists in shielding the molten weld puddle, thus minimizing oxidation of weld. Furthermore, a water/air recirculation is externally required for transferring heat from torch to the atmosphere. The nozzle feeding the plasma should be gentle and constituent, that it provides for welding of thin sheet, as well as fine wires. Such type of welding eliminates arc wander because there is no need to bias the electrode and the work piece. This type of welding can be used for components like needles, wires, light bulb filaments, thermocouples, probes, surgical instruments, die and mold repairs etc. (Henryk., 2011).

\subsection{Model Description}

The details of plasma production has been explained in (Tyagi et al., 2012) by using the KSF6 gas and potassium atoms (K) with the help of velocity shear instability controlled by electric and magnetic field. High-velocity plasma sources have been extensively applied in various fields of plasma physics, controlled thermonuclear fusion, plasma sputtering and plasma interaction with solid materials. Several methods have been suggested for producing a directed plasma jet of high velocity. The gun shown in Figure 1 is a coaxial plasma gun. The gun consists of plasma entrance nobe, inner and outer electrode, water circulation for cooling of nozzle and weld pool etc. The inner and outer diameters of the cylindrical electrodes are $6 \mathrm{~mm}$ and 14 $\mathrm{mm}$ respectively, with the lengths $120 \mathrm{~mm}$ and $125 \mathrm{~mm}$ correspondingly. Both electrodes are made of graphite. The two auxiliary spark plugs required to trigger the gun at the desired time are located 180" apart over the fast valve ports as shown in Figure 1. The gas filling is continuously adjustable from $5 \times 10^{-4}$ to $0.5 \mathrm{~Pa} \mathrm{~m}^{3}$, the opening time of the valve ranges from 20 to 800 ps (Shen et al., 1995).

The velocity of ions in plasma which contains Velocity Shear Instability is calculated with the help of real frequency of the surface wave in plasma. With the help of velocity of ions, plasma parameters are calculated and the effect of electric and magnetic field on plasma parameters will be discussed. We can calculate the group velocity $(v)\left(\partial \omega_{r} / \partial k\right)$ with the help of real frequency of surface wave (Tyagi et al., 2011).

Now the ion's velocity $v_{p}$ (assuming it to be equal to the group velocity of wave, i.e. as $\partial \omega_{r} / \partial k$ (Tyagi et al., 2012), and using the expression for the real frequency $\omega_{r}$ of the wave incident on the work-piece surface according to equation (15) from (Tyagi et al., 2011) can be written as: 
$\frac{\overline{\omega^{\prime}}}{\Omega_{i}}=-\frac{b_{1}}{2 a_{1}}\left[1 \pm \sqrt{\left(1-\frac{4 a_{1} c_{1}}{b_{1}^{2}}\right)}\right]$

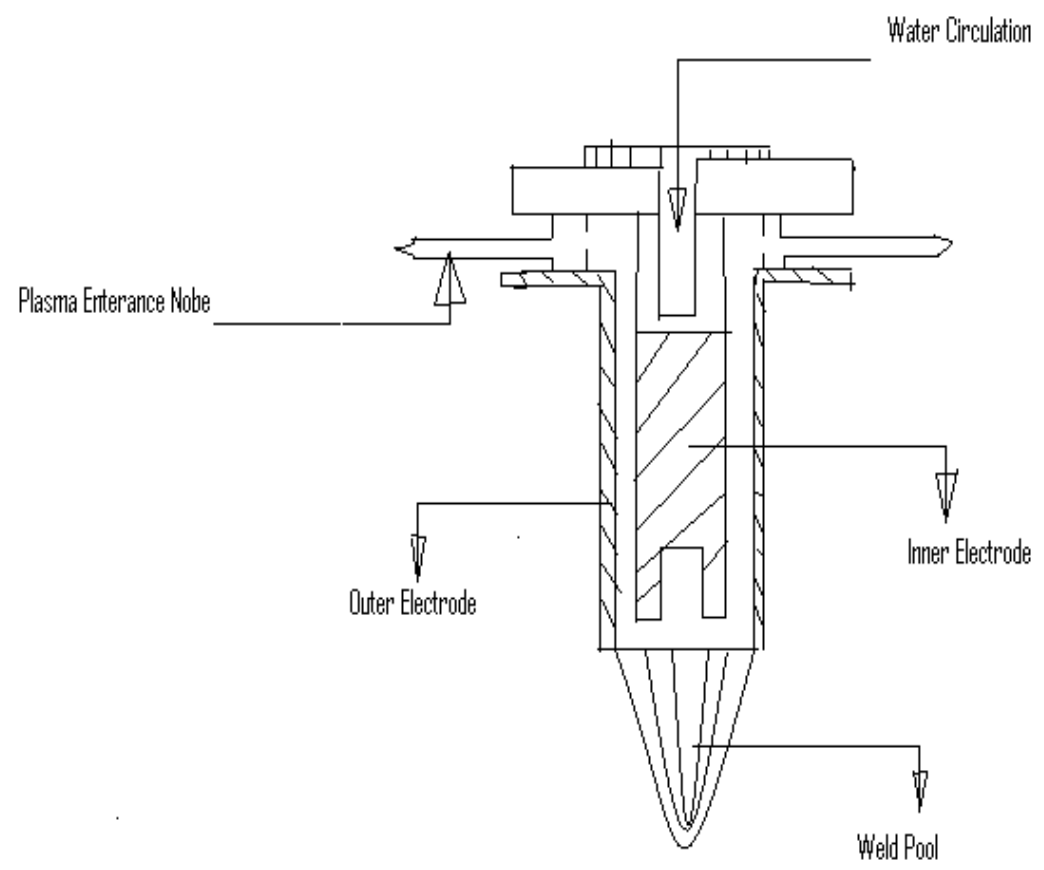

Figure 1. Model of Flasma Welding

From this expression, a dimensionless, real frequency has been calculated by computer technique when $b_{1}^{2}<4 a_{1} c_{1}$. Hence this criterion gives a condition for the real frequency of wave with homogeneous DC electric field, considering the inhomogeneity, $\left(\frac{x}{a}=0\right)$, in the electric field, to be neglected. This is a case of homogeneous DC electric field.

$$
\begin{aligned}
& a_{1}=a_{2}\left(\frac{\Omega_{i}}{k_{\| \mid} \alpha_{\|}}\right)^{2}, b_{1}=\frac{\Omega_{i}}{k_{\|} \alpha_{\| i}} b_{2}-\frac{2 k_{\perp} \Delta^{\prime}}{k_{\|}^{2} \alpha_{\| i}^{2}} a_{2} \Omega_{i} \\
& a_{2}=\frac{\eta_{e}}{\eta_{i}} \frac{T_{\perp} i}{T_{\| i}}+\frac{T_{\perp i}}{T_{\| i}}-\Gamma_{n}\left(\mu_{i}\right) \frac{T_{\perp i}}{T_{\| i}}, b_{2}=\frac{\Gamma_{n}\left(\mu_{i}\right) k_{\perp}}{2 k_{\|}} \varepsilon_{n} \rho_{i} \frac{\alpha_{\perp i}}{\alpha_{\| i}}-\frac{\Gamma_{n}\left(\mu_{i}\right) k_{\perp}}{2 k_{\|}}-\frac{\Gamma_{n}\left(\mu_{i}\right) k_{\perp} n \Omega_{i}}{2 k_{\|}^{2} \alpha_{\| i}} \\
& c_{1}=\frac{\Gamma_{n}\left(\mu_{i}\right) T_{\perp i}}{2 T_{\| i}}\left(1-\frac{k_{\perp}}{k_{\|}} A_{i}\right)-\frac{b_{2} k_{\perp} \Delta^{\prime}}{k_{\|} \alpha_{\| i}}+\frac{k_{\perp}^{2} \Delta^{\prime 2}}{k_{\|}^{2} \alpha_{\| i}^{2}} \\
& \eta_{i}=1-\frac{\overline{E_{i}}(x)}{4 \Omega_{i}^{2}}, \eta_{e}=1-\frac{\overline{E_{e}^{\prime}}}{4 \Omega_{e}^{2}}, \overline{\omega^{\prime}}=\bar{\omega}-n \Omega_{\mathrm{i}} \\
& P=\frac{x^{2}}{a}, E(x)=E_{0 x}\left(1-\frac{x^{2}}{a^{2}}\right), \bar{E}(x)=\frac{e_{s} E(x)}{m_{s}}, E(x)=E_{0 x}\left(1-\frac{x^{2}}{a^{2}}\right)
\end{aligned}
$$




$$
\begin{aligned}
& \Omega_{s}=\frac{e_{s} B_{0}}{m_{s}} ., \alpha_{\perp s}=\left(\frac{2 k_{B} T_{\perp s}}{m_{s}}\right)^{1 / 2}, \quad \alpha_{\| s}=\left(\frac{2 k_{B} T_{\mid s}}{m_{s}}\right)^{1 / 2} \\
& \xi=\frac{\bar{\omega}-(n+p) \Omega_{s}-k_{\perp} \Delta^{\prime}}{k_{\|} \alpha_{\| s}}, \Delta^{\prime}=\frac{\partial \Delta}{\partial t}, \Delta=\frac{\bar{E}(x) t}{\Omega_{s}}\left[1+\frac{E^{\prime \prime}(x)}{E(x)} \cdot \frac{1}{4}\left(\frac{v_{\perp}}{\Omega_{s}}\right)^{2} \ldots \ldots \ldots \ldots . . .\right. \\
& A_{s}=\frac{1}{\Omega_{s}} \frac{\delta v_{o z}(x)}{\delta x}, \varepsilon_{n}=\frac{\delta \ln n_{0}(x)}{\delta x}, A_{T}=\frac{\alpha_{\perp s}^{2}}{\alpha_{\| s}^{2}}-1, \bar{\omega}=\omega-k_{\| \mid} v_{o z}(x) \\
& \Gamma_{n}\left(\mu_{s}\right)=\exp \left(-\mu_{s}\right) I_{n}\left(\mu_{s}\right),
\end{aligned}
$$

Now energy of ions converted to thermal energy is:

$e V p=K_{b}{ }_{i}=m v p^{2} / 2$

Where $v_{p}$ is the group velocity of waves/ions found out by equation (1), $\mathrm{m}$ is the mass of ions, $\mathrm{K}_{\mathrm{B}}$ is the Boltzman constant, $T_{\mathrm{i}}$ is the temperature of ions.

$$
v_{p}=\frac{\text { real frequency of waves }}{k_{\amalg}} .
$$

By Tse et al. (1999) Debye length, number of ions striking the probe are written as:

$$
\lambda_{D e}=\sqrt{\varepsilon_{0} T_{e} / / e n_{0}}
$$

$\lambda_{D_{e}}$, is the Debye length of ion, $n_{0}$ is the plasma density.

$$
n_{\text {probe }}=\frac{n_{0} V_{p}}{4} \exp \left(\frac{e V_{p}}{K_{b} T_{e}}\right)
$$

$n_{\text {probe }}$, is the numbers of ions striking the probe per unit area per second

\section{Result and Discussion}

Numerical investigation with the help of mathematical formulation and computer technique by using experimental data such as electric and magnetic field, density gradient, temperature anisotropy etc (Kim and Merlino, 2007) has been analyzed for equations (1), (2), (3) and (4).The generated plasma have $\mathrm{SF}_{6}^{-}$ions in majority. In this section, we find the solution of equations (1), (2), (3), (4) using parameters like magnetic field, density gradient, thermal velocities, temperature ratio etc. It is assumed that electron and ion temperature ratio

$$
\frac{T_{e}}{T_{i}} \text { is } 1 \text { and magnetic field strengths are varying from } 0.24 \text { to } 0.28 T \text {, homogeneous DC electric field strength from } 16 \mathrm{~V} / \mathrm{m}
$$
to $24 \mathrm{~V} / \mathrm{m}$, in which ion temperature anisotropy

$$
A_{T}=\frac{T_{\perp i}}{T_{\| i}}-1=0.5 \text { with density gradient } \varepsilon_{n} \rho_{i}=0.2 \text {, parallel velocity shear with scale length } A_{i}=0.5 .
$$

The above selected values are experimental parameters which have been taken from Kim and Merlino (2007) and others parameters are assumed and suitable for our calculations. The relation between number of ion-acoustic waves, temperature of ions, Debye length, and electron heat flux is given by (Bychenkov and Silin, 1981) as:

$$
N(k)=\frac{26 q_{e}}{k T_{i}} \frac{\left(m_{e} m_{i}\right)^{1 / 2}}{k^{4}} \ln \left(\frac{1}{k \lambda_{D e}}\right) \delta \delta\left(\cos -\cos \theta_{0}\right)
$$


$N(k)$ is number of ion waves, $q_{e}$ is heat flux, $r_{D e}$ is Debye length, $\theta$ is angle between the wave vector $k$ and the heat flux, $\theta_{0}=145^{0}$.

The relation between heat flux and number of ions striking the target, temperature of ions/electron, ion acoustic wave velocity is given by Bychenkov and Silin (981) as:

$q_{e} \geq 6.5 n_{e} k T_{e} v_{s}$

Equations (5) and (6) show that heat flux in plasma welding increases by increasing the value of Debye length, temperature of ions and number of ions/electrons on the working surface, which indicates that the above features are predominant parameters in plasma welding. Figure 2 shows the variation of temperature of ions in $\mathrm{K}$ versus electric field in $\mathrm{V} / \mathrm{m}$. Other parameters are listed in figure caption.

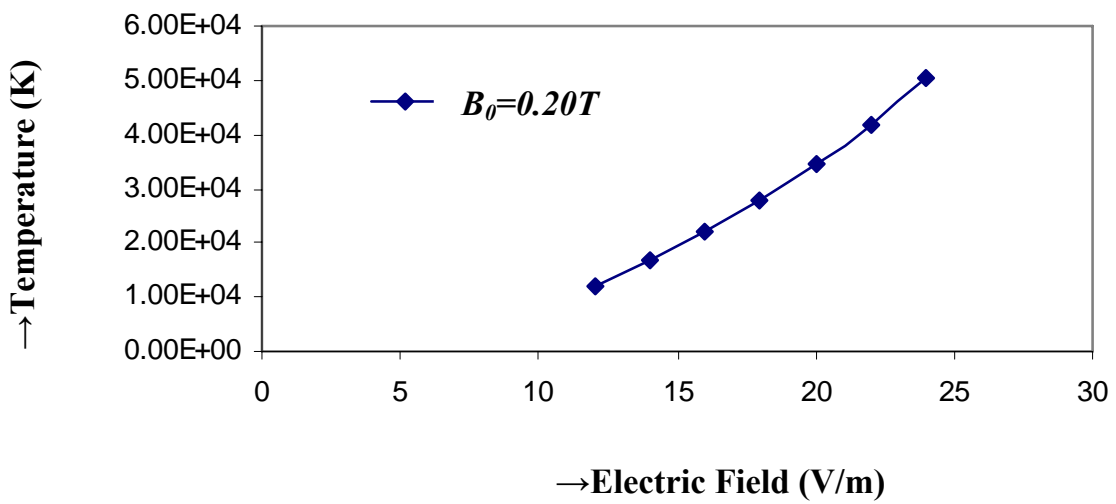

Fig.2.Variation of temperature verses electric field and other parameters are

$$
A_{i}=0.5, T_{e} / T_{i}=1, \theta_{1}=88.5^{0}, A_{T}=0.5, \varepsilon_{n} \rho_{\mathrm{i}}=0.2, k_{\perp} \rho_{i}=2 .
$$

Temperature increase by the increase of electric field is because of the change in acceleration of ions and wave. The effect of electric field is governed by the mathematical expression

$$
\frac{e E_{O}}{m_{i}} \mathrm{~m} / \mathrm{sec}^{2} \text {. }
$$

The temperature changes from $1 \times 10^{4} \mathrm{~K}$ to $5.02 \times 10^{4} \mathrm{~K}$ by changing the electric field $12 \mathrm{~V}$ to $24 \mathrm{~V}$ respectively. Figure 3 . shows the variation of Debye length in $(\mathrm{mm})$ versus electric field in volts. Other parameters are defined in figure caption. The Debye length has been increased by increasing the electric field. It changes from $2.5 \times 10^{-6} \mathrm{~mm}$ to $5.5 \times 10^{-6} \mathrm{~mm}$ by changing the electric field from $12 \mathrm{~V}$ to $24 \mathrm{~V}$, at a temperature of $5.02 \times 10^{4} \mathrm{~K}$. Debye length is the ratio of thermal velocity of ions and gyro frequency of ions. The thermal velocity is affected by temperature.

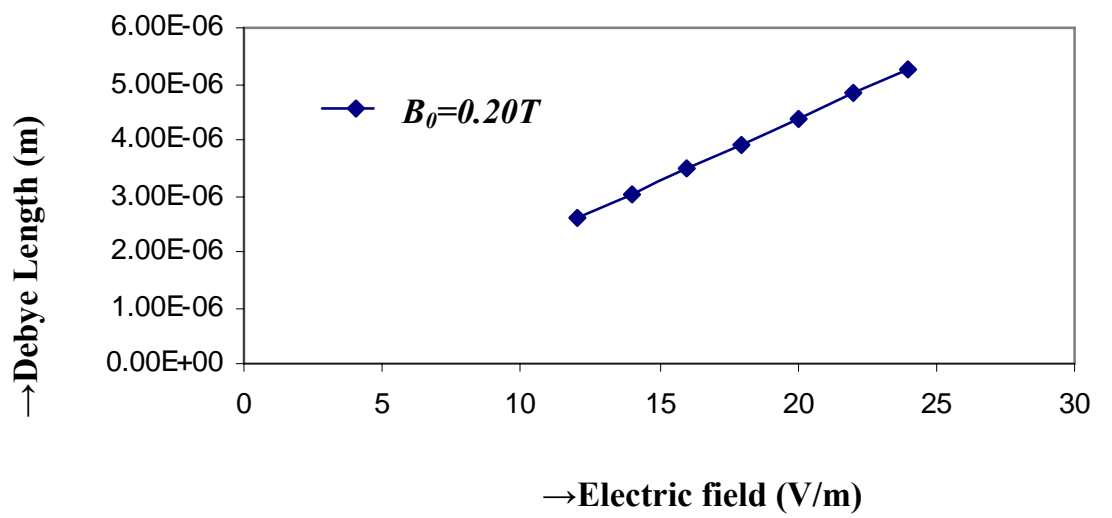

Fig.3. Variation of Debye Length verses Electric field and other parameters are $A_{\mathrm{i}}=0.5, T_{e} / T_{i}=1, \theta_{1}=88.5^{0}, A_{T}=0.5, \varepsilon_{n} \rho_{i}=0.2$,

$$
k_{\perp} \rho_{i}=2 .
$$


In Figure 4, the number of ions striking the probe per second versus electric field has been shown and other parameters being fixed are also shown in figure caption. The number of ions striking the probe has been increased by increasing the electric field. It will change from $3.5 \times 10^{25}$ per second to $7.0 \times 10^{25}$ per second by changing the electric field from $12 \mathrm{~V}$ to $24 \mathrm{~V}$. Figure (5), (6), (7) shows variation of ion temperature, Debye length, number of ions striking the probe per unit area per second versus magnetic field when varied from $0.120 T$ to $0.28 T$. Maximum value of ion temperature, Debye length, number of ions striking the probe are 1.75 $\mathrm{X} 10^{5} \mathrm{~K}, 2.25 \times 10^{-5} \mathrm{~mm}$, and $1.27 \times 10^{26}$ respectively when $\mathrm{k}_{\perp} \rho_{\mathrm{i}}=2$, with other parameters being listed in figure caption. The effect of magnetic field on temperature, Debye length and number of ions striking the probe per unit area per second has been decreased by increasing the magnetic field.

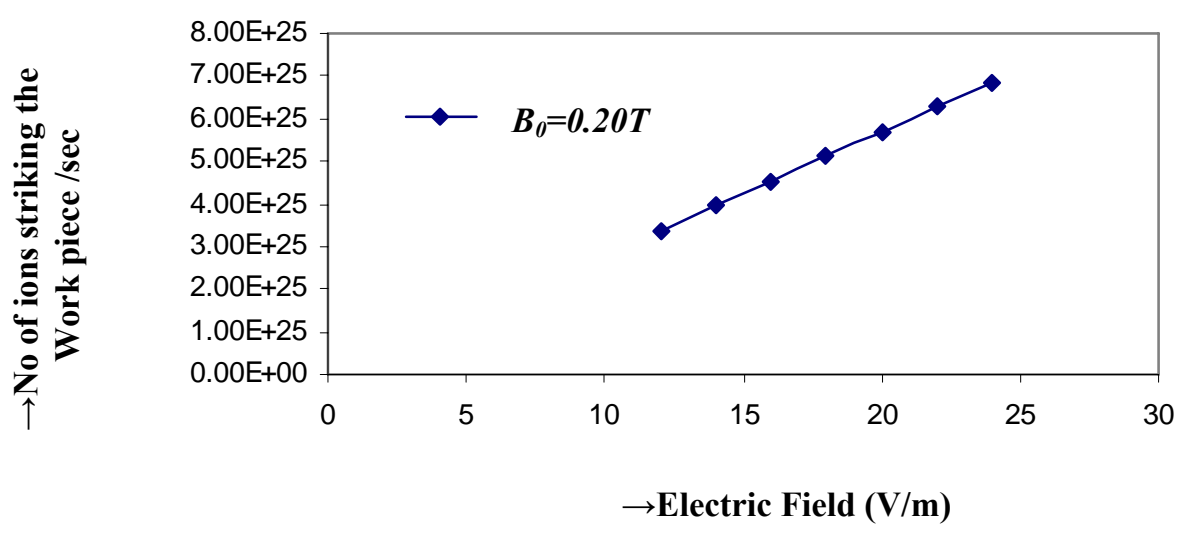

Fig.4. Variation of number of ions striking the probe verses Electric field and other parameters are $A_{i}=0.5, T_{e} / T_{i}=1, \theta_{1}=88.5^{0}$,

$$
A_{T}=0.5, \varepsilon_{n} \rho_{i}=0.2, k_{\perp} \rho_{i}=2 .
$$

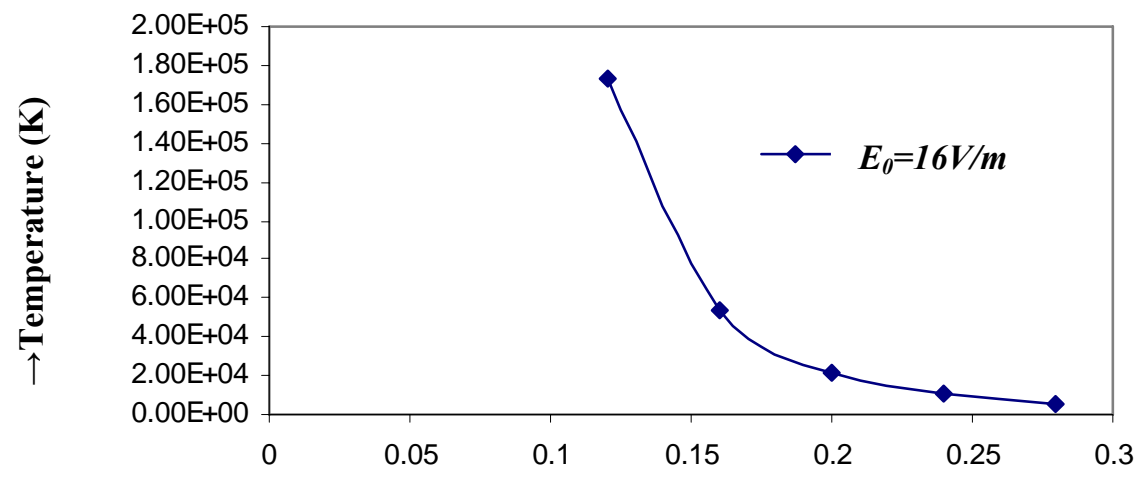

$\rightarrow$ Magnetic Field (T)

Fig.5. Variation of temperature verses magnetic field and other parameters are $A_{i}=0.5, T_{e} / T_{i}=1, \theta_{1}=88.5^{0}, A_{T}=0.5, \varepsilon_{n} \rho_{i}=0.2$,

$$
k_{\perp} \rho_{i}=2 .
$$

The plasma Debye length is experimentally found out by ( Paradkar et al., 2011) in which Debye length are in a range of $10^{-6} \mathrm{~m}$, when density of plasma varies from $10^{18} / \mathrm{cm}^{3}$ to $10^{26} / \mathrm{cm}^{24}$. The value of Debye length calculated by mathematical formulation and computer technique is also in the range of experimental values. The plasma temperature experimentally found by (Bourham and Gilligan., 1996) in which temperature of plasma species varies from $8.8 \times 10^{3} \mathrm{~K}$ to $1.4 \times 10^{4} \mathrm{~K}$, the density of plasma is $10^{23} / \mathrm{m}^{3}$ The value of temperature of plasma species calculated by mathematical formulation and computer technique is also in the range of experimental values. 


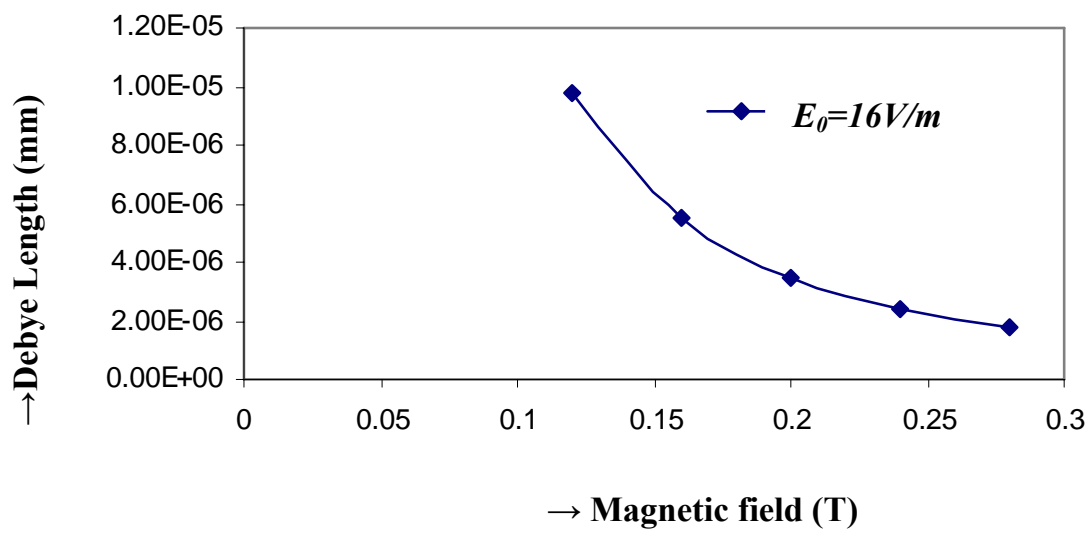

Fig.6. Variation of Debye Length verses magnetic field and other parameters are $A_{i}=0.5, T_{e} / T_{i}=1, \theta_{1}=88.5^{0}, A_{T}=1.5, \varepsilon_{n} \rho_{i}=0.2$,

$$
k_{\perp} \rho_{i}=2 .
$$

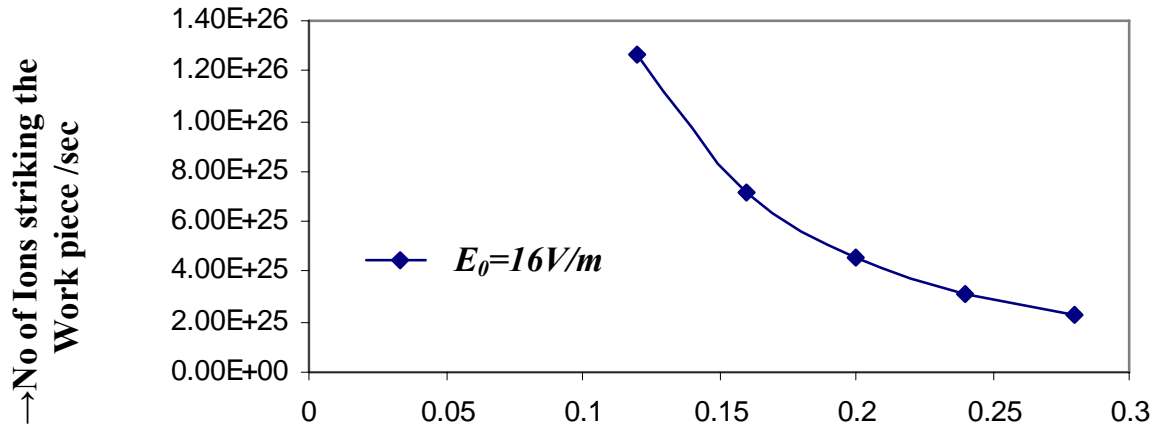

$\rightarrow$ Magnetic Field (T)

Fig.7.Variation of number of ions striking the probe verses Magnetic field and other parameters are $A_{i}=0.5, T_{e} / T_{i}=1, \theta_{1}=88.5^{0}$,

$$
A_{T}=0.5, \varepsilon_{n} \rho_{i}=0.2, k_{\perp} \rho_{i}=2 .
$$

\section{Conclusions}

This paper describes the mathematical model for plasma parameters. It shows the flexibility of using magnetic field and electric field to control Debye length, temperature of ions, and number of ions striking the work-piece. This work illustrates the effect of magnetic field and electric field on the number of ions striking the probe per unit area-second, by fixing the values of electron ion temperature ratio, temperature anisotropy of ions, shear scale length and density gradient on ions temperature, Debye length has been discussed separately. The results obtained will be useful for designing a new machine for plasma welding or to increase efficiency of existing machine. With the help of the above study, one can use a single plasma welding machine for different size/materials components by selecting appropriate plasma parameters.

Acknowledgement: I thank the reviewer for useful suggestions which have been incorporated at appropriate places, I am also thankful to Mr. Afthab Arafath Azeez for his contributions to the paper.

\section{Nomenclature}

$k_{I I}$

$k_{\perp}$

$\alpha_{11 i}$

$B_{0}$
Wave number in the direction of magnetic field

Wave number in the direction of electric field

Thermal velocity in magnetic field direction

Magnitude of magnetic field $(T)$ 


\begin{tabular}{|c|c|}
\hline$\Omega_{i}$ & Gyro frequency \\
\hline$\Gamma_{n}\left(\mu_{i}\right)=\exp \left(-\mu_{s}\right) I_{n}\left(\mu_{s}\right)$ & where $I_{N}\left(\mu_{s}\right)$ is modified Bessel function \\
\hline$T_{\perp i}$ & Temperature of ions in the direction of electric field \\
\hline$T_{\| i}$ & Temperature of ions in the direction of magnetic field \\
\hline$A_{i}$ & Shear Scale length of ions \\
\hline$x$ & Distance from origin \\
\hline$a$ & Gyro-radius of plasma waves \\
\hline$Z(\xi)$ & Plasma dispersion function \\
\hline$\omega=\omega_{r}+i \gamma$ & where $\omega_{r}=$ real frequency, $\gamma=$ growth rate \\
\hline$E(x)$ & Magnitude of homogeneous DC electric field w.r.t distance $\mathrm{x}$ from origin \\
\hline$E_{0 x}$ & Magnitude of homogeneous DC electric field at origin \\
\hline$e_{s}$ & Charge of species \\
\hline$m_{s}$ & Mass of Species \\
\hline$k_{B}$ & Boltzmann constant \\
\hline$n, p$ & Order of Bessel function \\
\hline$A_{s}$ & Shear Scale Length of species \\
\hline$\underline{\partial V_{o Z}(X)}$ & Velocity gradient \\
\hline$\partial X$ & \\
\hline$\varepsilon_{n}$ & Log mean plasma density gradient \\
\hline$\rho_{i}$ & Gyro-radius of ions \\
\hline$n_{0}(x)$ & Plasma density function of distance \\
\hline$A_{T}$ & Temperature anisotropy \\
\hline$q_{e}$ & Heat flux \\
\hline$\lambda_{D e}$ & Debye Length \\
\hline$\theta$ & Angle between the wave vector $\mathrm{k}$ and the heat flux \\
\hline$N(k)$ & Number of ion waves \\
\hline
\end{tabular}

\section{References}

Bourham M.A., Gilligan J.G.1996. Augmentation and control of burn rate in plasma devices. Contract N00014-95-1-1221, $P$ Number 96 pro 4536-00, pp.1-9.

Bychenkon Y.V., Silin V.P. 1981. Heat transfer in a turbulent laser plasma. JETP Lett Vol 34, No 6, pp.309-311.

Henryk M.S.2011. Properties comparison of joints made with $\mathrm{X}_{2} \mathrm{CrNi}_{12}$ ferrite steel sheets by plasma arc welding and spot resistance welding. Welding International, Vol 25, No 6, pp.425-429.

Kim S.-H., Merlino R.L.2007. Electron attachment to $\mathrm{C}_{7} \mathrm{~F}_{14}$ and $\mathrm{SF}_{6}$ in thermally ionized potassium plasma. Phys. Rev. E, Vol 76, No 3, pp. 035401(R) [4 pages].

Litovoko I.V. 2008. Computer modeling of extraction and transport of high-current charged particle beam. Cybernetics and Systems Analysis, Vol. 44, No. 5, pp.780-786.

Mannion M., Heinzman J. 1999. Plasma arc welding brings better control. Tooling and Production.

Nishiyama H., Saisu T., Okubo M., Kamiyama S. 1992. Numerical simulation of a low pressure plasma jet in the applied magnetic field. Institute of Fluid Science, Tohoku University Japan, pp. 284-289.

Paradkar B.S., Wei M.S., Yabuchi T., Stephens R.B., Haines M.G.,Krasheninnikov S.I., Beg F.N. 2011. Numerical modeling of fast electron generation in the presence of preformed plasma in laser-matter interaction at relativistic intensities. Physical Review E 83. 046401 No 4, pp. 1-10.

Shchitsyn Y.D., Shchitsyn Y.V.2003. Plasma welding of aluminum alloys. Welding International, Vol 17, No 1, pp.825-832.

Shen Z.G., Liu C.H., Lee C.H., Cheng U.1995. A study of a coxial plasma gun. J. Phys. D Appl. Phys Vol 28, pp. 314-318. 
Tyagi R.K., Srivastava K.K., Pandey R.S.2011.Analysis of electrostatic ion-cyclotron instability driven by parallel flow velocity shear. Journal of Surface Engineering and Applied Electrochemistry, Vol 47, No 4, pp.370-377.

Tyagi R.K., Srivastava K.K., Pandey R.S.2012.Nontraditional machining process by means of velocity shear instability in plasma. Journal of Surface Engineering and Applied Electrochemistry, Vol 48, No 1, pp.64-68.

Tyagi R.K., Pandey R.S. 2011. Effect of Electric and Magnetic field on metal cutting by velocity shear instability, Journal of Emerging Trends in Engineering and Applied Sciences, Vol. 2, No.4, pp.693-697.

Tse H.C., Man H.C., Yue T.M.1999. Effect of magnetic field on plasma control during $\mathrm{CO}_{2}$ laser welding. Optics and Lasers Technology Vol 31, No 5, pp.363-368.

Tse H.C., Man H.C., Yue T.M.2000. Effect of electric field on plasma control during $\mathrm{CO}_{2}$ laser welding. Optics and Lasers Technology Vol 33, No 3, pp.181-189.

\section{Biographical notes}

Mr. R.K. Tyagi is PhD student and pursuing PhD from Department of Mechanical Engineering, Birla Institute of Technology Mesra, Ranchi India. He is also working as Assistant Professor in Amity University Noida India. He published several papers in referred national and international journals. His area of research is industrial applications of plasma.

Dr.R.S. Pandey is Assistant professor in the Department of Applied Physics, Amity University UP Noida, India. He published more than 40 papers in referred national and international journals. He had also presented several research articles in national and international conferences. His area of research is plasma physics.

Dr.Arbind Kumar is Head of the department in the Department of Mechanical Engineering, Birla Institute of Technology Mesra, Ranchi, India. He has long teaching experience. He published several papers in referred national and international journals. He had also presented several research articles in national and international conferences. His area of research is renewable energy.

Dr.K.K. Srivastava is professor in Central University Jharkhand, Ranchi India. He has long teaching experience. He published papers in referred national and international journals. He had also presented several research articles in national and international conferences. His area of research is fluid mechanics.

Received July 201

Accepted January 2012

Final acceptance in revised form May 2012 